\title{
Confidence Limits Associated With Values of the Earth's Magnetic Field Used for Directional Drilling
}

Susan Macmillan, British Geological Survey; and Steve Grindrod, SPE, Copsegrove Developments

This is an author-created text version of Macmillan, Susan and Steve Grindrod, 2010. Confidence Limits Associated With Values of the Earth's Magnetic Field used for Directional Drilling. SPE Drilling \& Completion, 25(2), 230-238. DOI: 10.2118/119851-PA.

\section{Summary}

This paper describes updated uncertainties for use with predicted geomagnetic parameters within magnetic Measurement-While-Drilling (MWD) survey tool error models. These models are used to define positional error ellipsoids along the wellbore, which assist in hitting geological targets and avoiding collisions with existing wellbores.

The declination, dip angle, and total field strength of the Earth's magnetic field are used with magnetic survey tools for surveying the wellbore. These values are often obtained from mathematical models such as the British Geological Survey (BGS) Global Geomagnetic Model (BGGM). As the Earth's magnetic field is continually varying with time, the BGGM is updated annually to maintain accuracy. However, a global predictive model cannot capture all sources of the Earth's magnetic field, which results in uncertainties of the predicted parameters. The Industry Steering Committee on Wellbore Surveying Accuracy (ISCWSA) published an MWD error model in 2000 (Williamson 2000). The geomagnetic field uncertainties that are part of this model were derived from work done by the BGS in the early 1990s. Since then, more accurate data from magnetic survey satellites have been introduced into the BGGM, and the uncertainty of the predicted geomagnetic field parameters has been reduced.

The original approach to deriving the uncertainties involved separating the various error sources in the magnetic field and assessing them individually. This paper uses a simpler approach where clean orientated magnetic down-hole data are simulated using geomagnetic observatory data. Spot absolute measurements of the magnetic field made at observatories around the world are adjusted for the crustal magnetic field to make them more representative of hydrocarbon geology. The adjusted observatory data are then compared with the predicted values from the BGGM to assess the uncertainty. The uncertainties do not fit a normal distribution, so they are expressed as limits for various confidence levels. They vary with location and, in their derivation, do not assume any underlying empirical error distribution. While they also vary with time, we provide time-averaged look-up tables which should be valid for as long as there are good quality satellite data on which to base global magnetic field models. Options to further reduce the uncertainties using data from local magnetic surveys (In-Field 
Referencing) and observatories (interpolation IFR) are also described.

The use of the revised geomagnetic uncertainty values in the MWD error model will reduce wellbore position uncertainty to reflect the increased accuracy from recent improvements in geomagnetic modeling. This is demonstrated using results for the ellipsoids of uncertainty output by an MWD error model for three standard ISCWSA well profiles.

\section{Introduction}

The Earth's magnetic field is a vector quantity, dependent on position and time, and may be expressed as the vector sum of the contributions from three sources: the field generated in the Earth's core, the crustal field from local rocks, and a combined external field from electrical currents flowing in the upper atmosphere and magnetosphere. Global models used in directional drilling, for example the BGGM, generally represent a combination of the main (i.e., internal, field, and the undisturbed external field arising from ever-present magnetospheric currents). The values computed for the Earth's magnetic field at a given location and time from the BGGM will differ from the actual values that would be measured because of a combination of model errors, crustal fields, and external fields. It is the magnitude of uncertainty that we investigate here.

Directional drilling and survey management companies have a requirement for accurate geomagnetic field estimates and knowledge of the associated uncertainties when magnetic survey tools are used in the surveying of wellbores. Magnetic survey tools measure the direction of the wellbore relative to the direction of the local geomagnetic field. In addition, the magnetic dip angle and total intensity of the geomagnetic field are required for use in algorithms that reduce the error caused by the magnetic field associated with the drillstring. The uncertainties are used to compute positional error ellipsoids along the wellbore to help hit the increasingly small geological targets and miss other wellbores.

\section{Background and Error Distributions}

In 1993, BGS investigated the uncertainty in BGGM geomagnetic field values (Macmillan et al. 1993). However, the estimates of the crustal and external field contributions in the overall uncertainties were biased towards the North Sea. In addition, the BGGM has been continuously updated since 1993 to take account of new data, magnetic survey satellite missions, and improved field modeling methodology. The 1993 study formed the basis of the geomagnetic error terms included in the ISCWSA MWD error model. The ISCWSA MWD error model is designed to quantify wellbore position uncertainty, and it remains in use today [see ISCWSA (2009) for details of revisions). While other error terms in the model (there are about 136) have been updated, the geomagnetic error terms remain those that were derived by Williamson (2000) from the original study by Macmillan et al. (1993). Another reason for the update of the geomagnetic error terms is that there are significant 
differences between the terms from the MWD error model and those from the original study. Table $\mathbf{1}$ lists the geomagnetic error terms in the ISCWSA error model.

For a Gaussian or normal distribution of errors, the 1standard deviation $(1 \sigma)$ error limit is equivalent to being $68.3 \%$ confident that the estimated value is within that amount of the true value. Additionally, for a Gaussian distribution, $2 \sigma$ is equivalent to being $95.4 \%$ confident, and $3 \sigma$ is equivalent to being $99.7 \%$ confident. Although Williamson (2000) states that no restrictive assumptions are made about the statistical distribution of measurement errors, all of the input error magnitudes are quoted as 1standard deviation $(1 \sigma)$ values. To obtain 95.4 and $99.7 \%$ confidence limits for the output values, the computed final error estimates are multiplied by 2 and 3, respectively. It is, unfortunately, the case that for any other distribution of errors 2 or 3 times $1 \sigma$ is not equivalent to being 95.4 or 99.7\% confident. This is particularly true for geomagnetic data, and to obtain the 95.4 and $99.7 \%$ confidence limits, it is necessary to actually calculate them. The preferred confidence level in the oil industry for well-planning purposes is that it is equivalent to $2 \sigma$ (i.e., 95.4\%), and it is this level that is generally presented in this paper. However, as different companies use different confidence levels the detailed results for the 68.3, 90, 95, 95.4, 99, and 99.7\% levels are available online from BGS (BGS, 2009) or Copsegrove Developments (ISCWSA, 2009).

\section{Data and Analysis Methodology}

In this study, we use geomagnetic observatory data to estimate BGGM uncertainty: this is the next best source of data short of clean magnetic data measured within a wellbore. We compute the difference between model predictions and observatory data that were not used in the construction of a given BGGM model.

We define a "BGGM year" as the year starting shortly after the release of a BGGM in May of each year. For example, the 2006 BGGM year runs from 2006.5 to 2007.5. The majority of dates entered for magnetic field computations using a given release of the BGGM are expected to be within this period. By looking at each revision of the BGGM separately, we can see how the overall performance has changed over the years.

General Approach. For each BGGM, there are two stages in deriving its confidence limits. First of all, we calculate the minute by minute differences between the observations and the BGGM at each available observatory and use these differences to estimate the limits. Secondly, we adjust the minute by minute differences to account for the crustal field at each observatory. This is necessary because some observatories are sited on volcanic islands, and the crustal field contributions to their data are not representative of the magnetic fields experienced at oil fields.

We investigate the temporal dependence of the confidence limits based on all of the differences in each BGGM year and the spatial dependence by computing the limits at each site using recent BGGM years when data 
from modern magnetic survey satellites are being incorporated into the models.

Data Sources. Geomagnetic observatory minute means are the main data used in this study; Fig. 1a shows the observatory locations. This means that in any given BGGM year in the time period 1995-2007, there are between 40 and 50 million data from a maximum of 134 observatories available for comparison with a given BGGM model. The resolution of the data is 0.1 arcminutes (approximately $0.002^{\circ}$ ) for declination $(D)$ and magnetic dip angle (I), and $1 \mathrm{nT}$ for total field intensity $(F)$. Measurement accuracy is close to the resolution but does vary from one observatory to another depending on instrument stability and absolute baseline control.

Two sources of data were used to estimate the crustal field. First, global repeat station and magnetic survey data from 1985 and onwards. These are all land based. Second, we used all local datasets gathered in the vicinity of oil fields for the purposes of in-field referencing (IFR). These are dominated by aeromagnetic surveys and marine magnetic surveys. The coverage provided is shown in Figs. $1 \mathrm{~b}$ and 1c.

\section{Determining the Crustal Field Contribution to the}

Uncertainties. The global survey dataset provided a total of over 9,000 observations. The vector observations were compared to the BGGM model values; the differences between the observed values and BGGM2008 values were assumed to be dominated by the crustal field because the time-varying external field is removed in the measurement and reduction process. As the probability distribution plots of these differences had unexpected bumps towards more extreme values, it was suspected that there was a number of poor-quality observations. These had to be rejected to avoid biasing the final results. The standard deviation $(\sigma)$ of the absolute value of the differences was calculated for each field component and any differences greater than $3 \sigma$ were rejected as outliers. This procedure resulted in about $31 \%$ of $D, 17 \%$ of $I$ and $20 \%$ of $F$ observations being rejected respectively before the confidence limits were calculated. The confidence limits caused by the crustal field derived from the global survey data are shown in Table 2.

The IFR estimates provided spot estimates of the crustal field at over 200 sites, mostly offshore. Each site represents a distinct oil field. We transform the local scalar data to obtain vector data (Williamson et al. 1998) and also downward continue them to the maximum expected drilling depth. These data are also considered free of contamination by time-varying external fields and along with the standard practice of employing a base station nearby to correct for these signals, network leveling is widely used to remove any remaining contamination. The set of IFR estimates was not winnowed in any way before the limits were calculated. The resultant limits are shown in Table 3.

The limits of the crustal magnetic field from these two sources of data are combined by averaging and are then used to adjust the minute-by-minute differences between 
the observatory data and the BGGM. The aim of the adjustment at each observatory is to make the results representative of those that the end user of the BGGM is likely to encounter. For example, some observatories are located on volcanic rocks and the local magnetic field is therefore highly anomalous. These locations have very different geological settings compared to those of typical oil fields.

The adjustment is done by first making independent estimates of the observatory crustal field contribution. The observatory crustal biases are included as coefficients in an inversion of satellite and observatory data for a magnetic field model. This model is similar to the BGGM but with many more coefficients included to characterize the external field variations. Secondly, these estimates are subtracted from the differences. The differences are then ordered and the limits computed. Thirdly, these limits are combined with the new (smaller) crustal field limits by squaring, summing, and square rooting.

\section{Results}

The results for the $95.4 \%$ limits are presented according to how they vary with time, then with location.

Variations in Uncertainties With Time. The global limits for the $95.4 \%$ confidence level for each BGGM year is shown in Fig. 2. It can be seen that the limits are decreasing with time. This is because of the increase in the number of high-quality satellite data, especially since 2000 when Ørsted data were first incorporated into the BGGM.
There have also been various improvements in model parameterization and inversion techniques since 1995, but again especially since 2000. Because of these incremental improvements in the BGGM from one year to the next, any solar cycle effects are masked in the analysis presented here. Magnetic activity is linked with the approximately 11-year solar cycle, which had its last sunspot maximum during 2000 (with magnetic activity peaking in 2003) and last minimum during 2008.

The variations in the external field contributions to the 68.3, 95.4, and the $99.7 \%$ limits during a typical BGGM year at Eskdalemuir (relevant for drilling in the North Sea) are illustrated in Fig. 3. From this we can see that for high confidence levels, the BGGM is less accurate around about the equinoxes (March and October) when the alignment of the Earth's magnetic field in relation to the Sun's magnetic field carried in the solar wind is favorable for efficient transfer of solar wind energy into the magnetosphere. As a result, current systems within the magnetosphere and ionosphere are energized more during these periods. For low confidence levels (e.g., 68.3\%), the BGGM is less accurate during northern hemisphere summer when the ever-present auroral electrojet and midlatitude ionospheric dynamo current system are enhanced. It is important to realize that the limits in Fig. 3 are the external field contributions to the overall BGGM limits only: they have not been combined with the values from Tables 2 and 3. When this is done, the variations in time of the external field contribution are mostly saturated by the 
internal field contribution although the baseline contribution remains important.

There are also variations in the limits with time of day. This is illustrated in Fig. 4, again using Eskdalemuir data. From this we can see that for high confidence levels, the BGGM is less accurate (again the limits in Fig. 4 are the external field contributions only and have not been combined with the values from Tables 2 and 3) during the night when substorm processes in the tail of the magnetosphere are particularly prevalent. Instabilities in the tail plasma sheet of the magnetosphere occur, and the energy stored there is released when the magnetic field lines relax from their stretched, tail-like configuration and "snap" back into a more dipolar configuration. This process results in charged particles in the plasma sheet being energized and accelerated down to the polar ionosphere. However, for low confidence levels the midlatitude ionospheric dynamo current system is energized during sunlit hours, and, as a result, the BGGM is less accurate during these periods.

Fig. 5 shows how the $D$ limits compare with one another for each year, and from this, it can be seen that the $95.4 \%$ limits (equivalent to $2 \sigma$ if Gaussian) are more than twice the $68.3 \%$ limits (equivalent to $1 \sigma$ if Gaussian) and that the $99.7 \%$ limits (equivalent to $3 \sigma$ if Gaussian) are more than three times the $68.3 \%$ limits. This clearly demonstrates that we are dealing with a non-Gaussian error source.
Variations in Uncertainties With Location. The limits vary depending on location of the oil field. This is because of the external field contribution to the limits and the associated core field modeling challenges at locations where the external fields are significant. It is assumed that there are no significant spatial patterns in the crustal field contributions to the limits. However, it is conceivable that the crustal field is more significant at locations where the core field is strongest (i.e., at high latitudes, because part of the crustal field arises from magnetization that is induced by the core field). We expect any variations caused by changing inducing field strength to be small if they are detectable. The variations of the limits with location for BGGM2004 can be seen in Fig. 6.

As one might expect, the limits are higher at high latitudes where either the magnetic field lines are open to the solar wind and come under its influence or the auroral current systems are present. Charged particles of origin external to the Earth's magnetosphere can be accelerated down the open field lines and energize auroral and polar current systems. These particles can interact with atmospheric molecules and produce stunning auroral displays. The auroral electrojets are not centered on the geographic poles but on the geomagnetic poles. They are oval shaped, extending further from the geomagnetic poles and covering wider zones on the nightside than on the dayside. They vary in intensity according to time of day and also expand and contract according to activity. The BGGM limits, especially for $F$ in the region of the dip equator where the main magnetic field is horizontal, are 
also larger. As the dip equator is not well defined by geomagnetic coordinates based on a tilted dipole, we use corrected geomagnetic (CGM) coordinates (Gustafsson et al. 1992; Tsyganenko et al. 1987) to order the limits. CGM coordinates of a point in space are computed by tracing the magnetic field line through the specified point to the dipole geomagnetic equator, then returning to the same position along the dipole field line and assigning the obtained dipole latitude and longitude as the CGM coordinates to the starting point. At the near-equatorial region, where the magnetic field lines may not reach the dipole equator and where, therefore, the standard definition of CGM coordinates fails, a different approach based on a minimum intensity value along the given magnetic field line is applied. Essentially, CGM coordinates are better than straightforward geomagnetic coordinates (based on tilted dipole) because they take account of the higher spherical harmonic terms in the estimation of the main magnetic field.

Fig. 7 shows how the $95.4 \%$ limits vary with CGM latitude for BGGM2000-BGGM2006. Also plotted are splines (cubic B splines with 10 knots) that are used to interpolate between the CGM latitudes of the observatories. Organizing the limits in this manner allows one to see the effects of the auroral and equatorial currents more clearly in the results. While there are some unrealistic features in the results, for example the hemispherical asymmetry for the magnetic dip angle results, we choose to use them as they are rather than manipulate them by some arbitrary means to compensate for the inadequacies of observatory coverage at low latitudes and in the southern hemisphere. The expected increase in the $F$ limits in close vicinity of the dip equator is also missing because there are very few observatories directly affected by the equatorial electrojet at the moment. However, a larger-scale feature in the $F$ limits at low latitudes is present, and this is likely to be associated with the equatorial Appleton Anomaly (Lühr et al. 2003).

These spline functions are then used to derive timeaveraged look-up tables arranged by geographic latitude and longitude for each confidence level. The ISCWSA error model is in the process of being upgraded to have the option to read these tables. The tables can also be used to make global plots of the spatial variation of a particular limit and in Fig. 8, the 95.4\% limits are shown. The tables are available from BGS (2009) and ISCWSA (2009).

\section{Comparisons With Existing Magnetic Field}

Uncertainty Estimates. It is interesting to see how these estimates compare with those in the present ISCWSA error model, as detailed in Table 1. Fig. 9 shows the differences at the $95.4 \%$ confidence level in the sense (ISCWSA estimate - new estimate). In deriving these differences, we compute the $95.4 \%$ confidence limits as 2 times the $1 \sigma$ values as given in Table 1 , including of course the $B_{H}$ dependence for declination. This is the only method available for deriving these limits from the existing published literature (Williamson 2000). The new geomagnetic error estimates are a considerable 
improvement on the existing estimates. The same applies for the $68.3 \%$ confidence level.

\section{Implementation and Well Profile Comparisons Using}

Revised Magnetic Field Uncertainty Estimates. The geomagnetic error terms in the ISCWSA error models (Table 1) were updated using values from the look-up tables to derive new wellbore positional uncertainties for three typical well profiles. Specifically, tests were conducted using the standard MWD [Rev. 2] and MWD + Axial Correction [Rev. 2] models and the three standard ISCWSA well profiles: ISCWSA\#1 extended-reach well in the North Sea, ISCWSA\#2 fish-hook well in the Gulf of Mexico, and ISCWSA\#3 designer well in the Bass Strait (Williamson 2000). The standard MWD model assumes a magnetically clean drillstring whereas the MWD + Axial Correction model corrects for drillstring magnetization using magnetic dip and total intensity estimates.

The magnetic field error magnitudes are reduced to $1 \sigma$ equivalents by first extracting the limits for the location of the well and for the final confidence level desired, then dividing them by the equivalent number of $\sigma$ if it is assumed that the error distribution is Gaussian. This is so that the magnetic field errors can be combined and propagated with other error terms expressed as standard deviations. The divisors are 1 for the $68.3 \%$ limits, 1.64 for the $90 \%$ limits, 1.96 for the $95 \%$ limits, 2 for the 95.4\% limits, 2.58 for the $99 \%$ limits, and 3 for the $99.7 \%$ limits. For example, for ISCWSA\#1 well profile at $60^{\circ} \mathrm{N}$, $2^{\circ} \mathrm{E}$ and a $95.4 \%$ confidence in the final error ellipsoids, the standard geomagnetic error terms $A Z(G)$, $\mathrm{DBH}(\mathrm{G})$, $\operatorname{MFD}(G)$, and $\operatorname{MFI}(G)$ (Table 1) were replaced with $0.375^{\circ}, 0^{\circ}, 0.12^{\circ}$, and $96 \mathrm{nT}$. These are derived from the values in the $95.4 \%$ look-up table for $60^{\circ} \mathrm{N}, 2^{\circ} \mathrm{E}$, then dividing by 2 .

The error models are then run and the ellipsoid parameters at total depth and at the confidence level desired are output. These are compared with those from the existing error models. For ISCWSA\#3 well the comparison is at $3,000 \mathrm{~m}$ measured depth (MD) not the total depth at 4,030 $\mathrm{m}$ because the axial correction fails at $90^{\circ}$ hole inclination. Fig. 10 summarizes the results for lateral uncertainty (radius of the ellipsoid major axis). The percentage reduction is highest for low levels of confidence and lowest for high levels of confidence. For the $95.4 \%$ level the percentage reduction varies from $2 \%$ for ISCWSA\#2 well profile and the standard MWD model, to $27 \%$ for ISCWSA\#1 well profile and the MWD + Axial Correction model.

\section{Conclusions and Discussion}

Revised uncertainties to be associated with magnetic field values computed from the BGGM have been derived. These uncertainties are more robust than any computed previously and should be incorporated into implementations of the ISCWSA error model as soon as possible via the look-up tables. They will be valid as long as good-quality satellite data are available to help build global magnetic field models. 
Options exist to further reduce the uncertainties using data from magnetic surveys local to the drilling site (IFR) to estimate the local crustal field and, additionally, from nearby observatories (interpolation IFR) to estimate the local external field. If the site is on land, it is relatively easy to make vector observations in the vicinity of the drilling site (but at a sufficient distance to ensure no contamination from the drilling rig). However, at sea this is a much greater challenge because of the difficulties in establishing azimuth of a vector magnetometer system on a moving survey platform and of removing the magnetic effect of that platform. However, using a combination of gyros, GPS, and accelerometers for attitude determination and performing $360^{\circ}$ turns at locations where the magnetic field strength is already known, the accurate estimation of the magnetic field vector at sea has been shown to be feasible (Lesur et al. 2004).

One drawback of direct measurements of the geomagnetic field vector can be that the poor data coverage (caused by the time taken to make vector observations) precludes making an extensive map of the crustal field and estimating the field at depth. However, it is relatively easy to make observations of the strength of the magnetic field, and, if made from an aircraft, relatively quick. An extensive area can be covered in a few days, providing sufficient data for potential field transformations, such as directional derivatives and downward continuation. This method has been validated using aeromagnetic data over the UK landmass and comparing the transformed data with vector observations made on the ground. Early work on this is reported in Williamson et al. (1998). IFR reduces the contributions from the crustal field to the uncertainties by amounts similar to those in Tables 2 and 3, although some uncertainty is added back in depending on measurement or transformation method.

While the crustal field presents a bias error for all MWD survey measurements in a given well and dominates the uncertainty associated with any global magnetic field model, the external field also presents a bias error but affects individual survey measurements or sequence of measurements taken within a few hours of one another. The external field can be much more significant in terms of amplitude than the crustal field, especially at high latitudes. In fact, the external field dominates the pattern in the BGGM limits seen in Figs. 6 through 8 . If the surveys are from a critical part of the wellpath, the effects of magnetic activity on drilling decisions can be crucial. For these reasons, techniques such as interpolation IFR have been developed to estimate the magnetic field downhole on a minute-by-minute basis incorporating data from nearby magnetic observatories (Reay et al. 2005; Bowe and McCulloch 2007). As the next maximum in solar activity is expected in 2011 and magnetic activity is expected to increase from now till a couple of years after this, the benefits of using real-time geomagnetic data for correcting downhole surveys will increase over the next few years. 


\section{Acknowledgments}

We would like to thank Allan McKay (formerly BGS, now Petroleum GeoServices) for his contributions at an early stage of this work. The scientists and institutes operating geomagnetic observatories and undertaking magnetic surveys around the world are thanked for their tireless efforts. Global standards and dissemination of observatory data benefit greatly from the efforts of Intermagnet and the World Data Centers. Global magnetic field models are also dependent on data from magnetic survey satellites, and the Danish-led Ørsted and German-led CHAMP science teams are thanked in particular. The local magnetic data around oil fields have come from a wide variety of sources, and we thank the survey companies Halliburton Sperry Drilling Services, Baker Hughes INTEQ, Schlumberger, and Tech21 for helping make some of them available. Finally, the 2 reviewers are thanked for their comments, which helped improve the manuscript.

\section{References}

Bowe, J. and McCulloch, S. 2007. The Value of Real-time Geomagnetic Reference Data to the Oil and Gas Industry. In Space Weather: Research towards Applications in Europe, No. 344, ed. J. Lilensten, Chap. 5.2, 289-298. Dordrecht, The Netherlands: Astrophysics and Space Science Library, Springer.

British Geological Survey (BGS). 2009. BGS Global Geomagnetic Model, http://www.geomag.bgs.ac.uk/bggm.html.
Gustafsson, G., Papitashvili, N.E., and Papitashvili, V.O. 1992. A revised corrected geomagnetic coordinate system for Epochs 1985 and 1990. J. Atmos. Terr. Phys. 54 (11-12): 1609-1631. doi:10.1016/00219169(92)90167-J.

ISCWSA. 2009. MWD Error Model Rev.3, http://copsegrove.com/MWDModel.aspx.

Lesur, V., Clark, T., Turbitt, C., and Flower, S. 2004. A technique for estimating the absolute vector geomagnetic field from a marine vessel. J. Geophys. Eng. 1 (2): 109-115. doi:10.1088/1742-2132/1/2/002.

Lühr, H., Rother, M., Maus, S., Mai, W., and Cooke, D. 2003. The diamagnetic effect of the equatorial Appleton anomaly: Its characteristics and impact on geomagnetic field modeling. Geophys. Res. Lett. 30 (17): 1906. doi:10.1029/2003GL017407.

Macmillan, S., Firth, M.D., Clarke, E., Clark, T.D.G., and Barraclough, D.R. 1993. Error estimates for geomagnetic field values computed from the BGGM. Technical Report WM/93/28C, British Geological Survey, Keyworth, Nottingham, UK.

Reay, S.J., Allen, W., Baillie, O., Bowe, J., Clarke, E., Lesur, V., and Macmillan, S. 2005. Space weather effects on drilling accuracy in the North Sea. Annales Geophysicae 23 (9): 3081-3088.

Tsyganenko, N.A., Usmanov, A.V., Papitashvili, V.O., Papitashvili, N.E., and Popov, V.A. 1987. Software for Computations of the Geomagnetic Field and Related Coordinate Systems. Special Report, Soviet Geophysical Committee, Moscow, Russia. 
Williamson, H.S. 2000. Accuracy Prediction for Directional Measurement While Drilling. SPE Drill \& Compl 15 (4): 221-233. SPE-67616-PA. doi: 10.2118/67616-PA.

Williamson, H.S., Gurden, P.A., Kerridge, D.J., and Shiells, G. 1998. Application of Interpolation In-Field Referencing to Remote Offshore Locations. Paper SPE 49061 presented at the SPE Annual Technical Conference and Exhibition, New Orleans, 27-30 September. doi: 10.2118/49061-MS.

\section{Figure Legends}

Fig. 1-Locations of (a) observatories, (b) repeat stations, and (c) oil and gas fields with local magnetic data.

Fig. 2-The upper panel shows the $95.4 \%$ confidence limits in declination, magnetic dip angle (dip), and total intensity (B total) for BGGM1995-BGGM2006 and the lower panel shows the number of data used each BGGM year

Fig. 3-Variation by month of the external field contribution to the $68.3 \%$ (lower), $95.4 \%$ and $99.7 \%$ (upper) confidence limits in declination, magnetic dip angle and total intensity at Eskdalemuir in Scotland.

Fig. 4-Variation by hour of the external field contribution to the $68.3 \%$ (lower), $95.4 \%$ and $99.7 \%$ (upper) confidence limits in declination, magnetic dip angle and total intensity at Eskdalemuir in Scotland.

Fig. 5-The declination 68.3\% (lower trace), 90\%, 95\%, 95.4\%, 99\%, 99.7\% (upper trace) limits for BGGM1995BGGM2006

Fig. 6-Spatial variation in the 95.4\% limits for BGGM2004 in (a) declination, (b) magnetic dip angle, and (c) total intensity. Larger circle diameters indicate larger limits.

Fig. 7-The 95.4\% confidence limits in declination, magnetic dip angle, and total intensity for BGGM2000 2006 arranged by corrected geomagnetic latitude. Also shown are spline fits.

Fig. 8-Global 95.4\% limits for BGGM in (a) declination, (b) magnetic dip angle, and (c) total intensity. The grey areas in $8 \mathrm{a}$ are where declination is poorly defined.

Fig. 9-Differences in $95.4 \%$ confidence limits in the sense (ISCWSA estimate - new estimate) for (a) declination, (b) magnetic dip angle, and (c) total intensity. The grey areas in 9a are where the differences are off scale.

Fig. 10-Lateral position uncertainties at total depth for 4 ISCWSA error model [Rev. 2] implementations and 3 well profiles. 